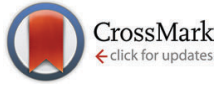

Cite this: J. Mater. Chem. C, 2016, 4, 1584

Received 4th January 2016

Accepted 20th January 2016

DOI: $10.1039 / \mathrm{c} 6 \mathrm{tc} 00026 f$

www.rsc.org/MaterialsC

\section{Efficient NIR light blockage with matrix embedded silver nanoprism thin films for energy saving window coatingt:}

\author{
M. Carboni, $\S^{a b}$ M. Carravetta, ${ }^{a}$ X. L. Zhang ${ }^{\star b}$ and E. Stulz ${ }^{\star a}$
}

\begin{abstract}
Highly efficient composite films, consisting of silica coated and functionalised silver nanoprisms (SNPs), are presented as a low-cost material to reduce thermal radiation flux with low impact on daylight transmission compared to similar solutions. The SNPs are covalently embedded in a poly(methyl methacrylate) (PMMA) matrix which prevents both leaking of the SNPs and aggregation within the matrix, thus enabling the formation of stable thin films with controllable dispersion. The cast thin films show absorbance in the IR region above $700 \mathrm{~nm}$ and a figure of merit of around 1, which is achieved with very thin films and low material consumption.
\end{abstract}

\section{Introduction}

Windows are regarded as one of the weakest elements in buildings with regard to insulation, leading to a rather high energy consumption due to either heating or cooling of the interior space. Although improvements have been achieved in reducing the heat transfer through convection and conductions, reducing heat transfer without hampering the light income is still challenging. In this communication, we report a composite film made of silica coated and functionalised triangular silver nanoprisms (SNPs) which are covalently embedded in a poly(methyl methacrylate) (PMMA) matrix as a low-cost material to reduce thermal radiation flux with low impact on daylight transmission.

The energy demand for residential buildings will show an average growth of approximately $0.6 \%$ every year until 2040 according to the latest Annual Energy Outlook. ${ }^{1}$ Projections show that this increase will be due to cooling rather than

\footnotetext{
${ }^{a}$ School of Chemistry \& Institute for Life Sciences, University of Southampton, Highfield, Southampton SO17 1BJ, UK. E-mail: est@soton.ac.uk

${ }^{b}$ Engineering and the Environment, University of Southampton, Highfield, Southampton SO17 1BJ, UK. E-mail:XL.Zhang@soton.ac.uk

$\dagger$ Raw analytical data are available from the authors upon request.

\$ Electronic supplementary information (ESI) available: Synthetic procedures and full characterisation of the composites. See DOI: 10.1039/c6tc00026f

$\S$ Current address: Building Energy Materials and Components, EMPA, 8600 Dübendorf, Switzerland.
}

heating as a result of the higher portion of urbanisation in warmer climate zones. The interest in technologies that can reduce the consumption of energy for heating or cooling buildings and vehicles therefore is steadily growing. In particular, the glazing of buildings offers great potential for energy saving as these account for about $30 \%$ of the total energy loss due to their high $U$-value $\left(\sim 0.8 \mathrm{~W} \mathrm{~m}^{-2} \mathrm{~K}^{-1}\right)$ compared to other elements of the building envelope. Currently, the research has focused on reducing the heat exchange, which is induced by radiative transfer, through direct coating of the glass. So far reflective coatings made of metal or metal oxide films are the materials of choice in commercial applications, but concern emerges regarding their extensive use in urban areas, particularly the formation of heating islands due to the reflection of the infrared radiation. ${ }^{2}$ The use of absorbing materials, on the other hand, is still limited due to the high impact on the visible light income through the windows. Although "smart" devices which are able to switch from a bleached (colourless) state to a coloured state are known (such as electrochromic or thermochromic materials), ${ }^{3-6}$ some issues still limit their use, which include durability, high material costs, switching time and deposition methods. ${ }^{7,8}$

The use of metal nanoparticles (MNPs) as dyes for energy saving coatings is gaining interest as these materials show strong extinction (absorption and scattering) due to the surface plasmon resonance (SPR), and show no photo-bleaching. Although MNPs usually show extinction in the visible part of light, their unique characteristics allow to precisely control the resonance wavelength. Particle size, shape and surrounding media are among the factors affecting the spectral position and the number of peaks. ${ }^{9,10}$ By modifying one or more of these parameters, the extinction maximum can be shifted to lower energy, and eventually near infrared radiation can be absorbed without affecting the visible light transmission. In principle, due to the high extinction coefficient of MNPs, high absorbance can be achieved through low concentration of particles, which effectively reduces the cost for solar control coating. Xu et al. ${ }^{11}$ have calculated that the cost of coatings made using gold nanorods dispersed in a poly(vinyl alcohol) (PVA) matrix (0.26 $\left.\mathrm{g} \mathrm{Au} \mathrm{m}^{-2}\right)$ 
would be of $\sim 3.76$ USD per $\mathrm{m}^{2}$ compared to the average price of $\sim 100$ USD per $\mathrm{m}^{2}$ of currently available technologies.

The pioneering work of Schelm et al. ${ }^{12,13}$ using $\mathrm{LaB}_{6}$ nanoparticles, which were dispersed in a poly(vinylbutyral) (PVB) matrix and sandwiched between two glass slides, demonstrated the feasibility of this approach. Reporting good figures of merit (FOM) in terms of the ratio between the visible and the solar transmitted light ( $T_{\text {lum }}$ and $T_{\text {sol }}$ ), the $\mathrm{LaB}_{6}$ particles outperform ITO based systems. NIR absorbing gold nanorods dispersed in a PVA matrix have recently been employed for window coatings by Stokes et al. ${ }^{14}$ These coatings absorb the NIR radiation up to $60 \%$ and show a good $T_{\text {lum }}$ of around $40 \%$.

Although these coatings are very promising, they still show some extinction in the visible spectrum, which could hinder their use in many applications such as glazing. Furthermore, simply dispersing the particles into a polymeric matrix could lead to aggregation of the particles, i.e. change in their optical properties and their leaching into the environment. Here we present the use of triangular silver nanoprisms (SNPs) as suitable materials for MNP based energy saving coating. ${ }^{15-20}$ We have chosen poly(methyl methacrylate) (PMMA) as a polymeric host as it does not show yellowing upon ageing, and due to its robustness under outdoor conditions; PMMA shows improved properties when doped with either silica ${ }^{21}$ or silver nanoparticles. ${ }^{22}$ The nanocomposites were prepared with different degree of polymerisation of PMMA and different SNP loading, and characterised using NMR and IR spectroscopy, GPC, TGA and TEM. The composites were deposited onto glass slides, and their efficiency in selectively absorbing the NIR portion ${ }^{22}$ of the spectrum without affecting the transmittance of the visible radiation was evaluated. To avoid aggregation of the metal nuclei in the polymeric host, SNPs were coated with a silica shell ( $\mathrm{SNP}_{\mathrm{SiO}}$ ) acting as an optically transparent protecting layer (Scheme 1). To create a stable dispersion of $\mathrm{SNP}_{2} \mathrm{SiO}_{2}$ in PMMA, the particles were further functionalised with allyl groups and polymerised with methyl methacrylate to form covalent $\mathrm{SNP}_{\mathrm{SiO}} /$ PMMA composites, which is a major step towards the formation of stable, non-leaching inclusion coatings. The solution was drop-cast onto the glass slides and the optical properties of the films were determined as the ratio of $T_{\text {lum }} / T_{\text {sol }}$. To our knowledge, we report the first covalently embedded polymer-NP

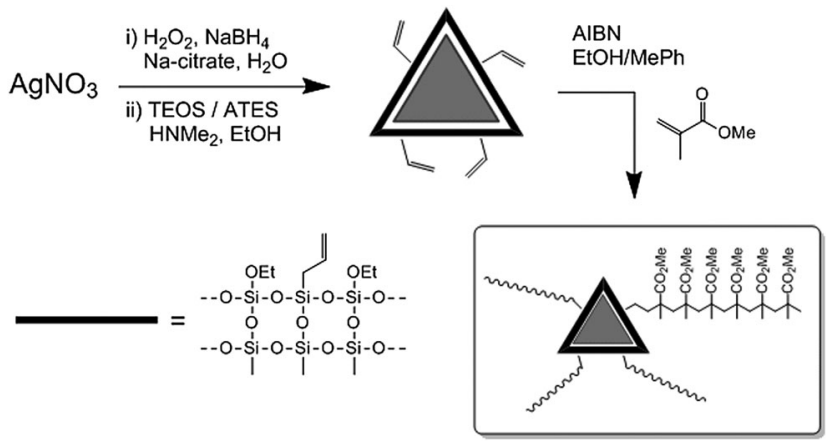

Scheme 1 Synthesis of the SNPaSiO $2 /$ PMMA composite. TEOS = tetraethoxy silane, ATES = allyl-triethoxy silane. composite with favourable optical properties suitable for window coatings; related systems include the formation of composites consisting of $\mathrm{Al}, \mathrm{Ag}, \mathrm{Au}, \mathrm{Cu}, \mathrm{Pt}, \mathrm{ZnO}$ and $\mathrm{Fe}$ nanoparticles embedded in polyacrylate, Teflon, polyvinyl(methyl ketone), poly(vinyl pyrrolidone), polyethylene, polydimethylsiloxane or PMMA.21,23-29

\section{Results and discussion}

The synthesis of the SNPs was conducted in bulk by adopting previously published methods ${ }^{20,30,31}$ and as described in the experimental section in the ESI.‡ In this way, the SNPs with an average size of $39 \pm 8 \mathrm{~nm}$ were obtained (SNP-1), according to TEM measurements (Fig. 1). Previous studies reported that chloride ions generally led to a increase in the size of silver nanoprisms. ${ }^{32}$ Addition of $\mathrm{KCl}$ prior to the reducing agent shows this effect also here, and the size of the SNPs increases to an average of $59 \pm 16 \mathrm{~nm}$ (SNP-2). This also has an impact on the optical properties of the SNPs (Fig. 1). While the smaller size SNP-1 shows an extinction maximum of around $620 \mathrm{~nm}$, the larger size SNP-2 shows a shift of the extinction to lower energy at around $750 \mathrm{~nm}$ with a concomitant lower extinction in the higher energy part of the visible spectrum, which is beneficiary for the anticipated application. The peak for SNP-2 also is broader due to sharper tips of the prisms, which was reported by Sherry et al. as a lightning rod effect. ${ }^{17}$ It is worth noting that a)

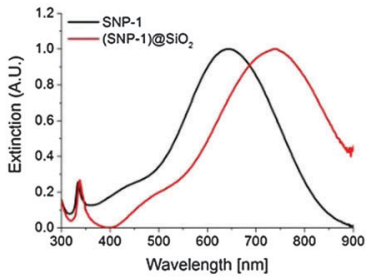

b)

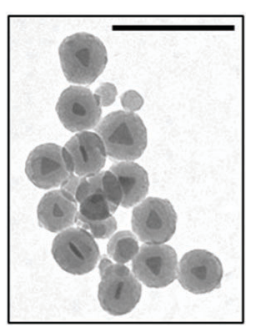

c)

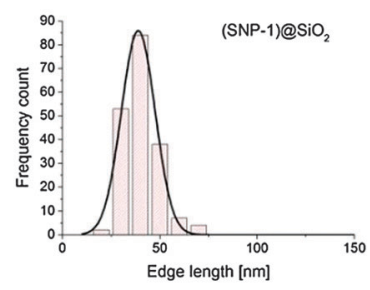

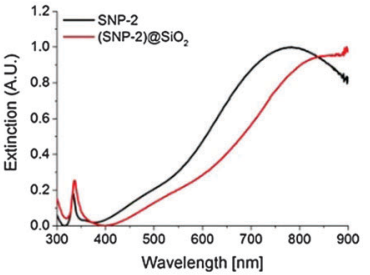
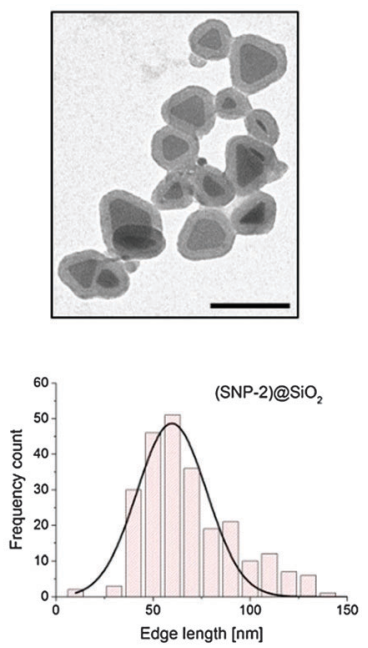

Fig. 1 Characterisation of the SNPs, showing (SNP-1)@SiO $\mathrm{S}_{2}$ in the left panel (prepared in the absence of chloride), and (SNP-2) $\mathrm{aSiO}_{2}$ in the right panel (prepared in the presence of chloride). (a) Extinction spectra of the SNPs, before (black line) and after (red line) coating with TEOS; (b) TEM images of $\mathrm{SNP} \mathrm{aSiO}_{2}$, scale bar $=200 \mathrm{~nm}$; (c) size distribution of SNPs obtained by the TEM measurements with the Gaussian fitting curve for average size determination. 
the extinction spectra of the MNPs are a result of a combination of absorption and scattering..$^{26}$ It is well understood that the contribution of scattering increases with the size of the MNPs, with the shape and surrounding environment also contributing. The contribution of scattering to extinction was calculated for triangular silver disks, where it was found that it is negligible for MNPs $<25 \mathrm{~nm}$, while it becomes an important factor for MNPs above $50 \mathrm{~nm}$, and is predominant for large MNPs of $>100 \mathrm{~nm}$ size. Scattering is in our case clearly not negligible, but absorption still remains the dominant contribution to the overall extinction.

The coating of nanoparticles with silica shells has been established with various metallic nanoparticles including silver, and has proven to be advantageous for MNPs coating due to its stability, transparency and availability for further functionalization through sol-gel chemistry. ${ }^{33-37}$ Adopting Mirkin's approach, ${ }^{35}$ the SNPs were first stabilized by surface functionalization with mercaptohexanoic acid, followed by growth of the silica shell in a TEOS solution using dimethylamine (DMA) as a catalyst to give $\mathrm{SNP} @ \mathrm{SiO}_{2}$. As reported previously, ${ }^{26}$ the coating with silica leads to a further red-shift by $\sim 70 \mathrm{~nm}$, which can be related to a shell thickness of approximately $15 \mathrm{~nm}$. The successful formation of the silica shell can be seen in the TEM images of both (SNP-1)@SiO ${ }_{2}$ and (SNP-2)@SiO ${ }_{2}$ (Fig. 1). To add functionality, $\mathrm{SNP} @ \mathrm{SiO}_{2}$ was formed in the presence of allyl-triethoxysilane (ATES). Co-condensation of TEOS with ATES was preferred over post-functionalization of the silica surface, which gave functionalization with better reproducibility. Using different TEOS/ATES ratios, the resulting silica shell becomes thinner due to shell growth blockage by ATES, leading to smaller red-shifts of the absorbance (see Fig. S1 in the ESI $\$$ for details). Energydispersive X-ray spectrum (EDAX) also shows strong reduction of $\mathrm{Si}$ counts compared to $\mathrm{Ag}$ counts upon addition of higher amounts of ATES (Fig. S1, ESI ). The IR spectrum of allyl-SNPs mainly shows signals due to the silicate shell, but weak peaks at $2919 \mathrm{~cm}^{-1}$ and $2850 \mathrm{~cm}^{-1}$, and at $1430 \mathrm{~cm}^{-1}$ are characteristic of the attached allyl-silane moiety (Fig. S2, ESI $\ddagger{ }^{38}$

The addition of allyl-groups to $\mathrm{SNP} @ \mathrm{SiO}_{2}$ is crucial for the formation of stable polymer inclusion systems through the covalent attachment of the SNPs to the polymer matrix; this also improves the dispersion of the SNPs within the matrix. To produce the SNP@SiO ${ }_{2} / \mathrm{PMMA}$ composites, the polymer was grown in the presence of various amounts of $\mathrm{SNP}_{\mathrm{SiO}}$ (see the $\mathrm{ESI} \ddagger$ for details). After polymerisation, the PMMA was precipitated in cold methanol, collected on a filter and was washed thoroughly with methanol. No SNPs were detected in the filtrate, showing the stable inclusion within the polymer matrix through grafting without leakage of the SNPs, which is a prerequisite to form useful long-lived window coatings. The IR spectrum of PMMA is not affected by the presence of the SNPs (Fig. S2, ESI $\ddagger$ ). To evaluate the effect of SNPs on the chain length of the polymer, PMMA was prepared with different AIBN concentrations $(1 \%, 2 \%$, $4 \%$ and $6 \%)$ in the presence of different amounts of allyl-SNP $(0,0.1 \%, 0.2 \%$ and $0.5 \% \mathrm{w} / \mathrm{w}$; Table $\mathrm{S} 1$ in the ESI $)$, and the average $M_{\mathrm{n}}$ and $M_{\mathrm{w}}$ of PMMA were determined using size exclusion chromatography (SEC). No clear trends can be observed upon addition of different amounts of allyl-SNPs, and only at $0.1 \%$ loading somewhat higher $M_{\mathrm{n}}$ and $M_{\mathrm{w}}$ values compared to polymerization in the absence of allyl-SNPs were seen. Since the lowest AIBN concentration (1\%) gave the longest polymer chains, further experiments were conducted under these conditions.

The SNP@SiO $/$ PMMA composites show a three-stage thermal degradation as determined by TGA, which is shown in Fig. 2 for SNP-1. The first two stages at $\sim 165{ }^{\circ} \mathrm{C}$ and $\sim 270{ }^{\circ} \mathrm{C}$, which have been assigned to the scission of the head-to-head linkages and to radical induced degradation, ${ }^{39,40}$ are largely unaffected by the presence of the SNPs. The third degradation event at $\sim 360{ }^{\circ} \mathrm{C}$, which is associated with random scission, shows a reduction of about $10{ }^{\circ} \mathrm{C}$ with increasing amount of allyl-SNP@SiO ${ }_{2}$. This is in contrast to what has been reported for bare spherical AuNPs ${ }^{22}$ and PdNPs ${ }^{41}$ which stabilise the PMMA matrix. Nevertheless, the loss in thermal stability is minimal under the expected working conditions. The covalent attachment of PMMA to the allyl-SNPs was also confirmed by NMR spectroscopy (Fig. 2). A sample of $6.5 \mathrm{~g}$ of the (SNP-1)@SiO 2 /PMMA composite (0.5\% SNP) was repeatedly washed with chloroform until no more unbound polymer was extracted, which required six washings. The majority of unbound polymer (99.4\%) was removed during the first washing (Fig. S3 and Table S2, ESI\$). Using magic-angle spinning (MAS) solid state NMR spectroscopy and ramped cross-polarization (CP) ${ }^{42}$ the ${ }^{13} \mathrm{C}$ signals of PMMA can clearly be recognised in the SNP@SiO ${ }_{2} / \mathrm{PMMA}$ composites. The small shifts of the signals of PMMA in the bound state are compatible with a covalent connection between the SNPs and PMMA.

Thin films of both (SNP-1)@SiO ${ }_{2} / \mathrm{PMMA}$ and (SNP-2)@SiO $/$ PMMA were prepared by slow evaporation of a chloroform suspension of the composites. The use of toluene or other solvents led to rather thick $(>1 \mathrm{~mm})$ and fragile films. The optimal concentration was found to be $20 \%$ of composites and produced films of $0.1 \mathrm{~mm}$ thickness with good visible transparency. The transmission spectra show strong absorbance at shorter wavelengths $(<1000 \mathrm{~nm})$ due to the SNPs (Fig. 3), where the peak for the SNP-2 system is broader and has a maximum at a higher wavelength of $880 \mathrm{~nm}$ compared to the SNP-1 system $(740 \mathrm{~nm})$; this is analogous the absorbance of the SNPs on their own.

To evaluate the efficiency of the composites as energy saving materials, the ratio between the transmittance of visible light $\left(T_{\text {lum }}\right)$ and the transmittance of total solar radiation $\left(T_{\text {sol }}\right)$ was a)

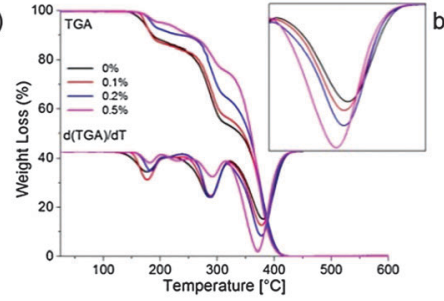

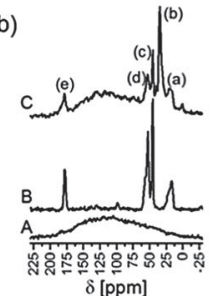

$\delta$ [ppm]
Fig. 2 (a) TGA and first derivative measured for the (SNP-1) @ $\mathrm{SiO}_{2} / \mathrm{PMMA}$ composite prepared with different SNP percentages $(0 \%, 0.1 \%, 0.2 \%$ and $0.5 \% \mathrm{w} / \mathrm{w}$ ) using $1 \% \mathrm{AIBN}$, the inset shows and expansion of the third stage; (b) CP MAS ${ }^{13} \mathrm{C}$ solid-state NMR spectrum of (SNP-1) $\mathrm{aSiO}_{2} / \mathrm{PMMA}$ after the removal of the physisorbed polymer by centrifugation and washing; A: (SNP-1) $\mathrm{aSiO}_{2}$, B: PMMA reference sample, C: (SNP-1) $\mathrm{CSiO}_{2} / \mathrm{PMMA}$. 

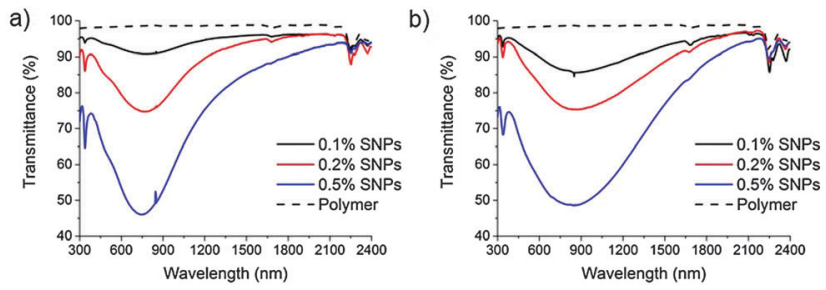

Fig. 3 UV-vis-NIR transmittance of thin films of $\mathrm{SNP} \mathrm{aSiO}_{2} / \mathrm{PMMA}$ prepared with SNP-1 (a) and SNP-2 (b), at $0.1 \%, 0.2 \%$ and $0.5 \%$ of SNP filler amounts, including pure polymer for comparison.

taken into account as FOM. The ideal value of the FOM would be 2.08 , corresponding to a material absorbing all the NIR radiation while passing all UV and visible light, but usually values above 1 are aimed at. ${ }^{14}$ The FOM values (Table 1 and Fig. S4, ESI $\ddagger$ ) are higher for the (SNP-2)@SiO $2 / \mathrm{PMMA}$ than for the (SNP-1)@SiO ${ }_{2} / \mathrm{PMMA}$ composites, with higher loading of SNP leading to a reduction of the FOM due to higher absorbance in the visible region. The best value was obtained for the system (SNP-2)@SiO $\mathrm{S}_{2}$ PMMA at 0.1\% SNP loading (FOM 1.011), showing that this system has characteristics very suitable for efficiently blocking the low energy light while not greatly disturbing the visual impact. This is particularly the case as the films are thin and contain a low loading of silver nanoprisms.

The FOM measured here is comparable to the FOM reported for gold nanorods (AuNRs) in PVA (1.000-1.030) where AuNRs were mixed into $15 \mathrm{wt} \%$ PVA solutions in various ratios, ${ }^{14}$ but is lower than for films prepared with $\mathrm{LaB}_{6}$ nanoparticles (FOM 1.705-1.934, 0.02-0.03\% NP loading). ${ }^{12}$ However, two considerations should be taken into account: firstly the reported films are $0.8 \mathrm{~mm}$ thick, and secondly different polymer matrices were used. Creating $0.8 \mathrm{~mm}$ films shows an improved FOM for (SNP-2)@SiO $\mathrm{S}_{2} / \mathrm{PMMA}$, which is maximum at $0.1 \% \mathrm{SNP}$ loading (1.063). At $0.2 \%$ SNP-2 loading some improvement in the FOM can also be seen, whereas for $0.5 \%$ SNP loading the films become visibly black with little light transmittance for both SNP-1 and SNP-2. In comparison, the correspondingly high

Table 1 FOM for NP composites with variable NP loading and film thickness

\begin{tabular}{|c|c|c|c|c|c|}
\hline Composite & $\begin{array}{l}\text { NP } \\
\text { loading }\end{array}$ & $\begin{array}{l}\text { Film thickness } \\
{[\mathrm{mm}]}\end{array}$ & $T_{\text {lum }}$ & $T_{\mathrm{sol}}$ & $\mathrm{FOM}^{a}$ \\
\hline \multirow[t]{5}{*}{ (SNP-1)@SiO $2 / \mathrm{PMMA}$} & \multirow[t]{2}{*}{$0.1 \%$} & 0.1 & 0.927 & 0.929 & 0.997 \\
\hline & & 0.8 & 0.410 & 0.416 & 0.985 \\
\hline & \multirow[t]{2}{*}{$0.2 \%$} & 0.1 & 0.816 & 0.830 & 0.982 \\
\hline & & 0.8 & 0.541 & 0.567 & 0.953 \\
\hline & $0.5 \%$ & 0.1 & 0.575 & 0.619 & 0.928 \\
\hline \multirow[t]{5}{*}{ (SNP-2)@SiO $2 / \mathrm{PMMA}$} & \multirow[t]{2}{*}{$0.1 \%$} & 0.1 & 0.909 & 0.899 & 1.011 \\
\hline & & 0.8 & 0.517 & 0.487 & 1.063 \\
\hline & \multirow[t]{2}{*}{$0.2 \%$} & 0.1 & 0.895 & 0.893 & 1.003 \\
\hline & & 0.8 & 0.463 & 0.453 & 1.022 \\
\hline & $0.5 \%$ & 0.1 & 0.586 & 0.601 & 0.975 \\
\hline \multirow[t]{2}{*}{ AuNR/PVA ${ }^{a}$} & Pane 1 & 0.8 & 0.37 & 0.37 & 1.000 \\
\hline & Pane 2 & 0.8 & 0.30 & 0.29 & 1.030 \\
\hline \multirow[t]{3}{*}{$\mathrm{LaB}_{6} / \mathrm{PVB}^{b}$} & $0.02 \%$ & 0.8 & 0.624 & 0.366 & 1.705 \\
\hline & $0.025 \%$ & 0.8 & 0.575 & 0.314 & 1.705 \\
\hline & $0.03 \%$ & 0.8 & 0.530 & 0.274 & 1.934 \\
\hline
\end{tabular}

${ }^{a}$ Data taken from ref. 13, NP loading not given. ${ }^{b}$ Data taken from ref. 11.
FOM for $\mathrm{LaB}_{6}$ is also influenced by the inherently high near-IR absorbance of the PVB matrix used. PVB has a transmittance of $\sim 70 \%$ in the visible light whereas PMMA is $100 \%$ transmitting, and the FOM of pure PVB was calculated to be $\sim 1.2$, which in itself is higher than the NP systems. In this respect, the use of thin PMMA films with low SNP loading shows great advantage over other films.

\section{Conclusions}

In summary, we have demonstrated efficient NIR light blockage of silver nanoprisms that are covalently incorporated into PMMA matrices to form thin film glass coating, whilst still being largely transmitting in the visible light region. The covalent embedding in the polymer matrix is a great advantage as it prevents both leaking of the NPs and aggregation within the matrix, thus enabling the formation of stable thin films with controllable dispersion. The polymerisation and the properties of the matrix are not significantly affected by the presence of the SNPs. Batch synthesis of SNPs in the presence of chloride followed by simultaneous silica coating and functionalization produces the most suitable particles. The cast thin films show absorbance in the IR region above $700 \mathrm{~nm}$, and a FOM which is comparable to other systems albeit achieved with much thinner films and lower material consumption. Even though some blockage of the visible light cannot yet be completely avoided, the system has a much lower impact on the perception of the light blockage. This should lay the foundation to further explore this system for the creation of suitable window coatings, which show reduced heat loss in buildings and vehicles, or indeed any other application where IR blockage is desired.

\section{Acknowledgements}

Financial support by ERDF (ISCE:Chem \& InterReg IVa program 4061) and an EPSRC DTC for Carboni is greatly acknowledged. Carravetta acknowledges the Royal Society for a University Research Fellowship.

\section{Notes and references}

1 Annual Energy Outlook 2015, U.S. Energy Information Administration, Washington, DC 20585, US, 2015.

2 N. J. Shih and Y. S. Huang, Build. Res. Inf., 2001, 29, 30-39.

3 S. Y. Li, G. A. Niklasson and C. G. Granqvist, J. Appl. Phys., 2014, 115.

4 C. G. Granqvist, P. C. Lansaker, N. R. Mlyuka, G. A. Niklasson and E. Avendano, Sol. Energy Mater. Sol. Cells, 2009, 93, 2032-2039.

5 E. Avendano, A. Azens, G. A. Niklasson and C. G. Granqvist, Sol. Energy Mater. Sol. Cells, 2004, 84, 337-350.

6 C. G. Granqvist, Sol. Energy Mater. Sol. Cells, 2000, 60, 201-262.

7 C. M. Lampert, Sol. Energy Mater. Sol. Cells, 2003, 76, 489-499.

8 N. A. O'Brien, J. Gordon, H. Mathew and B. P. Hichwa, Thin Solid Films, 1999, 345, 312-318. 
9 K. L. Kelly, E. Coronado, L. L. Zhao and G. C. Schatz, J. Phys. Chem. B, 2003, 107, 668-677.

10 W. Caseri, Macromol. Rapid Commun., 2000, 21, 705-722.

11 X. Xu, T. H. Gibbons and M. B. Cortie, Gold Bull., 2006, 39, 156-165.

12 S. Schelm, G. B. Smith, P. D. Garrett and W. K. Fisher, J. Appl. Phys., 2005, 97.

13 S. Schelm and G. B. Smith, Appl. Phys. Lett., 2003, 82, 4346-4348.

14 N. L. Stokes, J. A. Edgar, A. M. McDonagh and M. B. Cortie, J. Nanopart. Res., 2010, 12, 2821-2830.

15 D. Aherne, D. M. Ledwith, M. Gara and J. M. Kelly, Adv. Funct. Mater., 2008, 18, 2005-2016.

16 K. Munechika, J. M. Smith, Y. Chen and D. S. Ginger, J. Phys. Chem. C, 2007, 111, 18906-18911.

17 L. J. Sherry, R. C. Jin, C. A. Mirkin, G. C. Schatz and R. P. Van Duyne, Nano Lett., 2006, 6, 2060-2065.

18 R. Zmijan, M. Carboni, L. Capretto, E. Stulz and X. L. Zhang, RSC Adv., 2014, 4, 14569-14572.

19 M. Carboni, L. Capretto, D. Carugo, E. Stulz and X. L. Zhang, J. Mater. Chem. C, 2013, 1, 7540-7546.

20 G. S. Métraux and C. A. Mirkin, Adv. Mater., 2005, 17, 412-415.

21 J. de la Venta, A. Pucci, E. Fernández Pinel, M. A. García, C. de Julián Fernandez, P. Crespo, P. Mazzoldi, G. Ruggeri and A. Hernando, Adv. Mater., 2007, 19, 875-877.

22 V. V. Vodnik, J. V. Vukovic and J. M. Nedeljkovic, Colloid Polym. Sci., 2009, 287, 847-851.

23 F. Bauer, H.-J. Gläsel, U. Decker, H. Ernst, A. Freyer, E. Hartmann, V. Sauerland and R. Mehnert, Prog. Org. Coat., 2003, 47, 147-153.

24 A. Biswas, H. Eilers, F. H. Jr, O. C. Aktas and C. V. S. Kiran, Appl. Phys. Lett., 2006, 88, 013103.

25 N. Cioffi, N. Ditaranto, L. Torsi, R. A. Picca, E. D. Giglio, L. Sabbatini, L. Novello, G. Tantillo, T. Bleve-Zacheo and P. G. Zambonin, Anal. Bioanal. Chem., 2005, 382, 1912-1918.
26 D. Crespy and K. Landfester, Polymer, 2009, 50, 1616-1620.

27 A. Goyal, A. Kumar, P. K. Patra, S. Mahendra, S. Tabatabaei, P. J. J. Alvarez, G. John and P. M. Ajayan, Macromol. Rapid Commun., 2009, 30, 1116-1122.

28 S. Soumya, A. P. Mohamed, K. Mohan and S. Ananthakumar, Sol. Energy Mater. Sol. Cells, 2015, 143, 335-346.

29 J. L. Wilson, P. Poddar, N. A. Frey, H. Srikanth, K. Mohomed, J. P. Harmon, S. Kotha and J. Wachsmuth, J. Appl. Phys., 2004, 95, 1439-1443.

30 R. C. Jin, Y. C. Cao, E. C. Hao, G. S. Métraux, G. C. Schatz and C. A. Mirkin, Nature, 2003, 425, 487-490.

31 R. C. Jin, Y. W. Cao, C. A. Mirkin, K. L. Kelly, G. C. Schatz and J. G. Zheng, Science, 2001, 294, 1901-1903.

32 N. Cathcart, A. J. Frank and V. Kitaev, Chem. Commun., 2009, 7170-7172.

33 Y. Kobayashi, H. Katakami, E. Mine, D. Nagao, M. Konno and L. M. Liz-Marzan, J. Colloid Interface Sci., 2005, 283, 392-396.

34 L. M. Liz-Marzan, M. Giersig and P. Mulvaney, Langmuir, 1996, 12, 4329-4335.

35 C. Xue, X. Chen, S. J. Hurst and C. A. Mirkin, Adv. Mater., 2007, 19, 4071-4074.

36 S. H. Liu and M. Y. Han, Chem. - Asian J., 2010, 5, 36-45.

37 K. W. Shah, T. Sreethawong, S. H. Liu, S. Y. Zhang, L. S. Tan and M. Y. Han, Nanoscale, 2014, 6, 11273-11281.

38 Y. S. Chaudhary, J. Ghatak, U. M. Bhatta and D. Khushalani, J. Mater. Chem., 2006, 16, 3619-3623.

39 L. E. Manring, Macromolecules, 1991, 24, 3304-3309.

40 T. Kashiwagi, A. Inaba, J. E. Brown, K. Hatada, T. Kitayama and E. Masuda, Macromolecules, 1986, 19, 2160-2168.

41 C. Aymonier, D. Bortzmeyer, R. Thomann and R. Mullhaupt, Chem. Mater., 2003, 15, 4874-4878.

42 G. Metz, X. L. Wu and S. O. Smith, J. Magn. Reson., Ser. A, 1994, 110, 219-227. 